\title{
改进的抑制灰度单元的拓扑优化方法
}

\author{
高翔 ${ }^{1,2)}$, 王林军 $\left.{ }^{1,2}\right)^{*}$ ，杜义贤 ${ }^{1,2}$ \\ 1) (三峡大学水电机械设备设计与维护湖北省重点实验室 宜昌 443002) \\ 2) (三峡大学机械与动力学院 宜昌 443002) \\ (ljwang2006@126.com)
}

\begin{abstract}
摘＼cjkstart要：固体各向同性材料惩罚法或材料属性有理近似法所得拓扑优化结构中存在大量灰度单元，且现有 Heaviside 投影函数存在频繁调用密度矩阵的问题，进而导致计算效率较低. 针对此问题提出了一种基于改进 Heaviside 投影函 数的抑制灰度单元的拓扑优化方法. 首先, 建立以结构柔度最小化为设计目标的拓扑优化模型; 然后, 通过固体各 向同性材料惩罚法或材料属性有理近似法计算杨氏模量; 最后, 通过 Heaviside 投影函数抑制灰度单元，并由优化准 则法求解拓扑优化问题. 数值模拟结果表明，文中 2 个 Heaviside 投影函数能够稳定和有效地抑制灰度单元，且二者 分别对固体各向同性材料惩罚法和材料属性有理近似法所得结构的清晰度和刚度的改进效果比较明显.
\end{abstract}

关键词: 拓扑优化; 变密度法; Heaviside 投影函数; 抑制灰度单元

中图法分类号: TH122 DOI: 10.3724/SP.J.1089.2020.18208

\section{An Improved Topology Optimization Method for Suppressing Gray Elements}

\author{
Gao Xiang ${ }^{1,2)}$, Wang Linjun ${ }^{1,2)^{*}}$, and Du Yixian ${ }^{1,2)}$ \\ 1) (Hubei Key Laboratory of Hydroelectric Machinery Design and Maintenance, China Three Gorges University, Yichang 443002) \\ ${ }^{2)}$ (College of Mechanical and Power Engineering, China Three Gorges University, Yichang 443002)
}

\begin{abstract}
The topology optimization structure obtained by the SIMP (solid isotropic material with penalization) method or RAMP (rational approximation of material properties) method has a large number of gray elements, and the existing Heaviside function has the problem of calling the density matrix frequently, so the computational efficiency is relatively low. Aiming at this problem, an improved topology optimization method for suppressing gray elements is proposed in this paper. Firstly, a topology optimization model with minimizing the structural compliance as design objective is established; then, the Young's modulus is calculated by the SIMP method or RAMP method; finally, gray element is suppressed by the Heaviside projection function, and the topology optimization problem is solved by the OC (optimality criteria) method. The numerical simulation results show that: the two Heaviside projection functions proposed in this paper can suppress gray elements stably and effectively, and they can notably improve the definition and stiffness of the structure obtained by the SIMP method and RAMP method respectively.
\end{abstract}

Key words: topology optimization; variable density method; Heaviside projection function; gray element suppression

收稿日期: 2020-01-13; 修回日期：2020-09-22. 基金项目：国家自然科学基金(51775308); 湖北省水电机械设备设计与维护重点 实验室开放基金(2019KJX12). 高翔(1993一), 男, 硕士研究生, 主要研究方向为结构优化与可靠性分析、拓扑优化; 王林军(1982一), 男, 博士, 副教授, 硕士生导师, 论文通讯作者, 主要研究方向为结构优化与可靠性分析、反问题理论与方法; 杜义贤 (1978一), 男, 博士，教授，博士生导师，主要研究方向为拓扑优化、CAD/CAM 技术、结构优化与分析. 
为了使梁结构所用材料最少, 且满足结构的强 度要求, 需要对结构进行拓扑优化设计. Bendsø $\mathrm{e}^{[1]}$ 提出固体各向同性材料惩罚 (solid isotropic material with penalization, SIMP)法, Rietz ${ }^{[2]}$ 提出材料属 性有理近似(rational approximation of material properties, RAMP)法，且 Stolpe 等 ${ }^{[3]}$ 对 RAMP 法的收敛性 进行证明. Andreassen 等 ${ }^{[4]}$ 编写了一种高效的拓扑 优化代码，该代码包括确保稳定性的灵敏度过滤 方法、避免棋盘格现象的密度过滤方法，以及用于 三维拓扑优化或过滤半径过大的偏微分方程 (partial differential equation, PDE) 过滤器. Liu 等 ${ }^{[5]}$ 编写了一个关于 SIMP 的三维拓扑优化的 189 行 Matlab 代码. Zhu 等 ${ }^{[6]}$ 对柔顺机构的可靠性拓扑优 化和鲁棒性拓扑优化进行了研究, 并列举了大量 的拓扑优化代码. Wei 等 ${ }^{[7]}$ 提出一种基于径向基函 数的水平集拓扑优化方法, 并编写了 88 行的拓扑 优化代码. Yaghmaei 等 ${ }^{[8]}$ 基于 Matlab 内置的过滤函 数编写了 62 行的拓扑优化代码. $\mathrm{Gao}$ 等 ${ }^{[9]}$ 对多尺度 复合结构进行了研究, 并编写了相应的代码. Liu 等 ${ }^{[10]}$ 提出一种基于径向基函数(radial basis function, RBF)的水平集微结构拓扑优化方法.

Fuchs 等 ${ }^{[11]}$ 通过倒变量和(sum of the reciprocal variables, SRV)约束条件减少了结构中的灰度单 元. 吴一帆等 ${ }^{[12]}$ 提出 “阈值二分化”和“元胞平均 化”2 种方法, 它们均可得到清晰的拓扑优化结构. 张逸飞 ${ }^{[13]}$ 通过双向 Heaviside 函数实现了灰度单元 的抑制. 许小奎等 ${ }^{[14]}$ 针对灰度单元问题提出一种 密度体积插值方法, 在求解位移约束下体积最小 的拓扑优化问题中取得了良好的效果. 付永清等 ${ }^{[15]}$ 提出一种多层的密度更新规则, 减少了结构中的 灰度单元. 杜义贤等 ${ }^{[16]}$ 提出一种基于紧支 Shepard 近似的抑制灰度单元的方法. Guest 等 $^{[17]}$ 提出一种 向 0 极化的 Heaviside 投影函数，而 Sigmund ${ }^{[18]}$ 提 出一种向 1 极化的 Heaviside 投影函数, 且这 2 种 Heaviside 投影函数均可提高结构的清晰度. 龙凯 等 ${ }^{[19]}$ 提出 2 种 Heaviside 投影函数 ${ }^{[20-21]}$; Lee 等 ${ }^{[22]}$ 通过正则化理论构造了 4 种移动正则化 Heaviside 投影函数，且龙凯等 ${ }^{[19]}$ 和 Lee 等 ${ }^{[22]}$ 提出的向 0 和 1 两极分化的 Heaviside 投影函数均可获得清晰的边 界。杜义贤等 ${ }^{[23]}$ 针对灰度单元问题提出一种 Heaviside 投影函数，不仅可改变密度分界点，而 且可改变极化速率.

传统 Heaviside 投影函数 ${ }^{[17-23]}$ 存在以下问题: （1）投影函数中包含 2 项密度的一次项，或投影函
数的导数中包含密度的二次项, 会增加调用密度 矩阵的次数, 进而影响计算效率; (2) 无法抑制密 度为 0.5 的中间密度单元. 本文提出的 Heaviside 投影函数及其导数中，均仅有一个一次项，而且没 有二次项，将调用单元密度矩阵的次数减少 $50 \%$. 本文提出了 2 个改进的 Heaviside 投影函数, 为了 验证其通用性，将其分别应用于 SIMP 和 RAMP 这 2 种插值模型中, 并通过二维和三维算例对其性能 进行了对比, 在拓扑优化结构的清晰度和刚度方 面, 它们均优于采用传统 Heaviside 投影函数的拓 扑优化结构.

\section{1 拓扑优化模型}

连续体拓扑优化的优化模型为

$$
\left\{\begin{array}{l}
\min c\left(x_{e}\right)=\boldsymbol{U}^{\mathrm{T}} \boldsymbol{K} \boldsymbol{U}=\sum_{e=1}^{N} E_{e}\left(x_{e}\right) \boldsymbol{u}_{e}^{\mathrm{T}} \boldsymbol{k}_{0} \boldsymbol{u}_{e} \\
\text { s.t. } V\left(x_{e}\right) / V_{0}=f \\
\\
\boldsymbol{K} \boldsymbol{U}=\boldsymbol{F} \\
0 \leqslant x_{e} \leqslant 1
\end{array}\right.
$$

其中, $\boldsymbol{U}$ 为位移列向量; $\boldsymbol{K}$ 为刚度矩阵; $\boldsymbol{F}$ 为载 荷列向量; $x_{e}$ 为单元密度; $V\left(x_{e}\right)$ 为当前体积; $V_{0}$ 为初始体积; $f$ 为目标体积分数.

SIMP 法和 RAMP 法的杨氏模量插值计算公式 分别如下.

(1) SIMP 法的插值函数 ${ }^{[1]}$ 为

$$
E_{e}\left(x_{e}\right)=E_{\min }+x_{e}^{p}\left(E_{0}-E_{\min }\right)
$$

其中, $E_{\min }$ 为杨氏模量的下限; $E_{0}$ 为杨氏模量的上 限; $p$ 为惩罚因子.

SIMP 法中柔度的灵敏度为

$$
\frac{\partial c}{\partial x_{e}}=-\sum_{e=1}^{N} p x_{e}^{p-1}\left(E_{0}-E_{\min }\right) \boldsymbol{u}_{e}^{\mathrm{T}} \boldsymbol{k}_{0} \boldsymbol{u}_{e}
$$

(2) RAMP 法的插值函数 ${ }^{[2-3]}$ 为

$$
\frac{1}{E_{e}\left(x_{e}\right)}=\frac{1}{E_{\min }}+x_{e}\left(\frac{1}{E_{0}}-\frac{1}{E_{\min }}\right)
$$

如果令惩罚因子 $q=\left(E_{0}-E_{\text {min }}\right) / E_{\text {min }}$, 则插值 函数可转化为

$$
E_{e}\left(x_{e}\right)=E_{\min }+\frac{x_{e}}{1+q\left(1-x_{e}\right)}\left(E_{0}-E_{\text {min }}\right)
$$

RAMP 法中柔度的灵敏度为

$$
\frac{\partial c}{\partial x_{e}}=-\sum_{e=1}^{N} \frac{1+q}{\left[1+q\left(1-x_{e}\right)\right]^{2}}\left(E_{0}-E_{\min }\right) \boldsymbol{u}_{e}^{\mathrm{T}} \boldsymbol{k}_{0} \boldsymbol{u}_{e}
$$

SIMP 法和 RAMP 法中, 体积的灵敏度均为 


$$
\partial V\left(x_{e}\right) / \partial x_{e}=1
$$

式(2)(5)对应的函数曲线如图 1 所示.

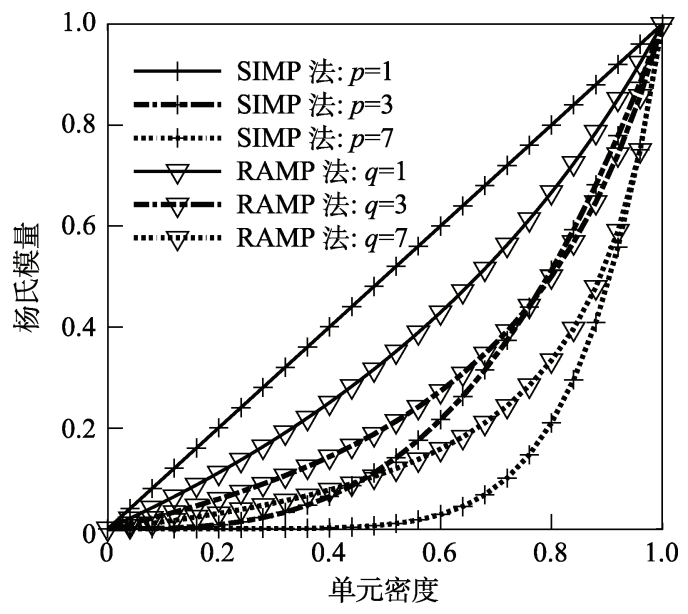

图 12 种方法的对比

由图 1 可知, 随着系数不断变化, RAMP 法的 插值曲线变化幅度小于 SIMP 法.

根据该拓扑优化数学模型，可建立拉格朗日 函数 ${ }^{[24]}$

$$
\begin{aligned}
& L\left(x_{e}\right)=c\left(x_{e}\right)+\lambda_{1}\left(V\left(x_{e}\right)-V_{0}\right)+\lambda_{2}^{\mathrm{T}}(\boldsymbol{K} \boldsymbol{U}-\boldsymbol{F})+ \\
& \lambda_{3}^{\mathrm{T}}\left(x_{1}-\boldsymbol{X}\right)+\lambda_{4}^{\mathrm{T}}(\boldsymbol{X}-1)
\end{aligned}
$$

其中， $\lambda_{1}, \lambda_{2}, \lambda_{3}$ 和 $\lambda_{4}$ 分别为拉格朗日乘子; $x_{1}$ 和 $x_{\mathrm{u}}$ 分别为单元密度的下界和上界, 取值分别为 0.001 和 1.000 .

如果采用 $\mathrm{OC}$ 法对密度进行更新, 需根据拓扑 优化模型建立对应的 Kuhn-Tucker 条件 ${ }^{[24]}$

$$
\left\{\begin{array}{l}
\frac{\partial c}{\partial x_{e}}+\lambda_{1} \frac{\partial\left[\frac{V\left(x_{e}\right)}{V_{0}}-f\right]}{\partial x_{e}}+ \\
\lambda_{2}^{\mathrm{T}} \frac{\partial(\boldsymbol{K} \boldsymbol{U}-\boldsymbol{F})}{\partial x_{e}}\left\{\begin{array}{l}
>0, \text { if } x_{e}=x_{1} \\
=0, \text { otherwise } \\
<0, \text { if } x_{e}=x_{\mathrm{u}}
\end{array}\right. \\
V\left(x_{e}\right) / V_{0}-f=0 \\
\boldsymbol{K} \boldsymbol{U}-\boldsymbol{F}=0 \\
\boldsymbol{\lambda}_{3} \geqslant 0, \lambda_{4} \geqslant 0
\end{array}\right.
$$

根据 Kuhn-Tucker 条件可求得密度的更新 公式 [4]

$$
x_{e}^{\text {new }}= \begin{cases}\max \left(0, x_{e}-m\right), & \text { if } x_{e} B_{e}^{\eta} \leqslant \max \left(0, x_{e}-m\right) \\ \min \left(1, x_{e}+m\right), & \text { if } x_{e} B_{e}^{\eta} \geqslant \min \left(1, x_{e}+m\right) \text { (10) } \\ x_{e} B_{e}^{\eta}, & \text { otherwise }\end{cases}
$$

其中，启发式更新因子 $B_{e}=\frac{-\partial c / \partial x_{e}}{\lambda \partial V / \partial x_{e}}$.

\section{2 抑制灰度单元的方法}

由于 SIMP 法和 RAMP 法的拓扑优化结果中 存在灰度单元, 本文依次使用密度过滤方法和 Heaviside 投影函数对灰度单元进行抑制.

(1) 密度过滤方法.

若采用密度过滤方法去除棋盘格现象，则过 滤以后的灵敏度和单元密度分别为

$$
\begin{gathered}
\frac{\partial \phi}{\partial x_{j}}=\sum_{e \in N_{j}} \frac{\partial \phi}{\partial \hat{x}_{j}} \frac{\partial \hat{x}_{j}}{\partial x_{j}}=\sum_{e \in N_{j}} \frac{1}{\sum_{i \in N_{e}} H_{e i}} H_{j e} \frac{\partial \phi}{\partial \hat{x}_{e}} \\
\hat{x}_{e}=\frac{1}{\sum_{i \in N_{e}} H_{e i}} \sum_{i \in N_{e}} H_{e i} x_{i}
\end{gathered}
$$

其中, 卷积算子 $H_{e i}=\max \left(0, r_{\min }-\Delta(e, i)\right) ; \quad N_{e}$ 代 表 $\Delta(e, i)<r_{\min }$ 的区域; $\hat{x}_{e}$ 为密度过滤以后的密度. 由于体积的敏度和柔度的敏度公式类似，所以此 处统一用 $\phi$ 表示, 避免重复的公式. $H_{e i}$ 和 $H_{j e}$ 均 为卷积算子, 而且分别对应 $i \in N_{e}$ 和 $e \in N_{j}$.

(2) Heaviside 投影函数.

若采用 Heaviside 投影函数抑制灰度单元，则 体积和柔度的灵敏度为

$$
\frac{\partial \phi}{\partial x_{j}}=\sum_{e \in N_{j}} \frac{\partial \phi}{\partial \hat{x}_{e}} \frac{\partial \hat{x}_{e}}{\partial x_{e}}=\sum_{e \in N_{j}} \frac{1}{\sum_{i \in N_{e}} H_{e i}} H_{j e} \frac{\partial \phi}{\partial x_{e}^{\text {new }}} \frac{\partial x_{e}^{\text {new }}}{\partial \hat{x}_{e}}(13)
$$

其中, $x_{e}^{\text {new }}$ 为 Heaviside 投影函数变换后的单元密度.

Guest 等 ${ }^{[17]}$ 提出的投影函数, 其表达式及其导 数分别为

$$
\left\{\begin{array}{l}
\text { 函数: } x_{e}^{\text {new }}=1-\exp \left(-\beta \hat{x}_{e}\right)+\hat{x}_{e} \exp (-\beta) \\
\text { 导数: } \frac{\partial x_{e}^{\text {new }}}{\partial \hat{x}_{e}}=\beta \exp \left(-\beta \hat{x}_{e}\right)+\exp (-\beta)
\end{array}\right.
$$

由于上述投影函数中含有 2 个密度的一次项, 因此单元密度矩阵需要参与 2 次计算. 由于设计域 被分割为大量的离散单元，而单元密度矩阵由大 量的单元密度 $\hat{x}_{e}$ 组成, 因此单元密度矩阵的维度 较大. 为了避免频繁地调用单元密度矩阵, 需要构 造一种 Heaviside 投影函数, 且该函数有且仅有一 项单元密度 $\hat{x}_{e}$ 的一次项. 本文提出的投影函数中 单元密度矩阵只需参与一次计算, 减少了调用单 元密度矩阵的次数, 从而提高了拓扑优化的计算 效率. 该投影函数及其导数分别为

$$
\left\{\begin{array}{l}
\text { 函数: } x_{e}^{\text {new }}=1-\frac{\exp \left[\beta\left(1-\hat{x}_{e}\right)\right]-1}{\exp (\beta)-1} \\
\text { 导数: } \frac{\partial x_{e}^{\text {new }}}{\partial \hat{x}_{e}}=\frac{\beta \exp \left[\beta\left(1-\hat{x}_{e}\right)\right]}{\exp (\beta)-1}
\end{array}\right.
$$


式(14)(15)的函数曲线如图 2 所示.

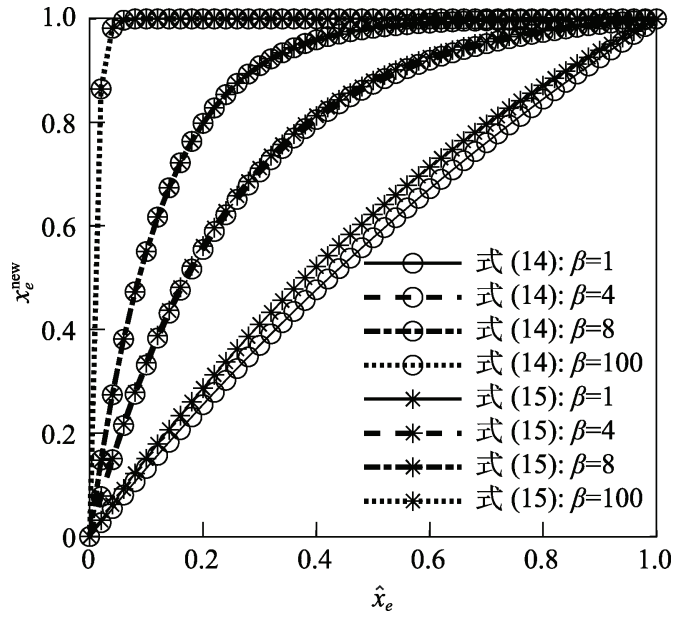

图 2 式(14)(15)的函数曲线

由图 2 可知, 本文提出的 Heaviside 投影函数 与式(14)的相似度极高. 此外, 式(15)及其导数中 仅含有一项密度的一次项. 所以，在密度更新和过 滤时，单元密度矩阵仅参与一次计算.

Sigmund ${ }^{[18]}$ 提出的投影函数, 其表达式及其导 数分别为

$$
\left\{\begin{array}{l}
\text { 函数: } x_{e}^{\text {new }}=\exp \left[-\beta\left(1-\hat{x}_{e}\right)\right]-\left(1-\hat{x}_{e}\right) \exp (-\beta) \\
\text { 导数: } \frac{\partial x_{e}^{\text {new }}}{\partial \hat{x}_{e}}=\beta \exp \left[\beta\left(\hat{x}_{e}-1\right)\right]+\exp (-\beta)
\end{array}\right.
$$

上述投影函数中含有 2 个密度的一次项. 当密 度更新时, 需要调用 2 次单元密度矩阵. 为了减少 调用密度矩阵的次数, 本文提出的投影函数的表 达式及其导数分别为

$$
\left\{\begin{array}{l}
\text { 函数: } \quad x_{e}^{\mathrm{new}}=\frac{\exp \left(\beta \hat{x}_{e}\right)-1}{\exp (\beta)-1} \\
\text { 导数: } \frac{\partial x_{e}^{\mathrm{new}}}{\partial \hat{x}_{e}}=\frac{\beta \exp \left(\beta \hat{x}_{e}\right)}{\exp (\beta)-1}
\end{array}\right.
$$

式(16)(17)的函数曲线如图 3 所示.

由图 3 可知, 式(16)(17)的相似度很高, 而且 式(17)中密度的一次项比式(16)少一项. 在密度更 新和过滤时, 单元密度矩阵仅参与一次计算.

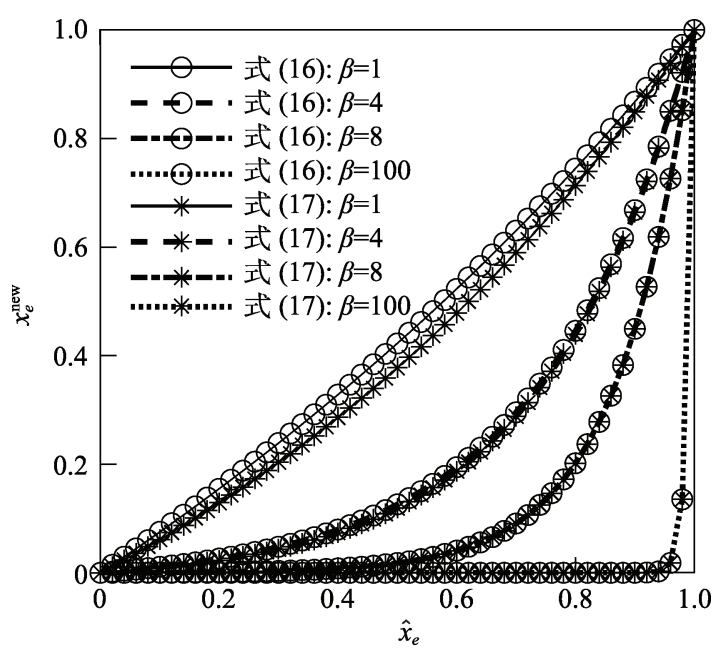

图 3 式(16)(17)的函数曲线

\section{3 算 例}

\section{1 二维简支梁的拓扑优化}

简支梁的左下角固定，右下角简支，离散单元 个数为 $120 \times 60$, 集中力作用在下平面的中心. 该 简支梁的结构示意图如图 4 所示.

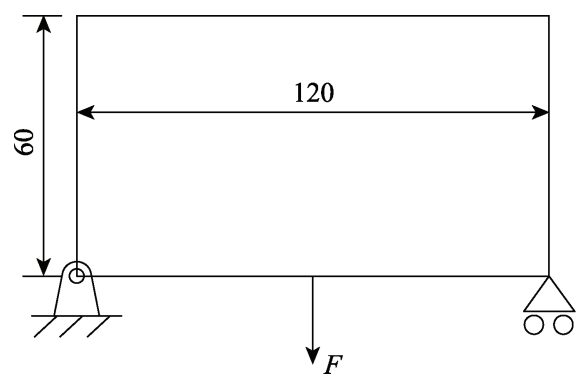

图 4 二维简支梁的结构示意图

设 $F=1, E_{\min }=1 \times 10^{-9}, E_{0}=1, f=0.50, r_{\min }=2.5$ ， $p=3, q=3$; 将 2 种投影函数式(14)(15)分别应用于 SIMP 法和 RAMP 法中. 参数 $\beta$ 的初始值均为 1 , 每 50 步乘以 2 , 且 $\beta$ 的最大值为 512.2 种 Heaviside 投影函数所得优化结构如表 1 所示, 柔度的计算迭

\begin{tabular}{|c|c|c|c|c|c|c|c|c|c|c|}
\hline \multirow{3}{*}{ 函数 } & \multicolumn{5}{|c|}{ SIMP 法 } & \multicolumn{5}{|c|}{ RAMP 法 } \\
\hline & \multicolumn{2}{|c|}{ 迭代/次 } & \multirow{2}{*}{ 柔度 } & \multirow{2}{*}{ 总时间/s } & \multirow{2}{*}{ 结构 } & \multicolumn{2}{|c|}{ 迭代/次 } & \multirow{2}{*}{ 柔度 } & \multirow{2}{*}{ 总时间 $/ \mathrm{s}$} & \multirow{2}{*}{ 结构 } \\
\hline & 外层 & 总数 & & & & 外层 & 总数 & & & \\
\hline 式(14) & 445 & 20761 & 14.4476 & 60.03 & & 489 & 23001 & 14.4637 & 74.23 & \\
\hline 式(15) & 479 & 22332 & 14.4385 & 61.44 & & 488 & 22925 & 14.6185 & 73.27 & \\
\hline
\end{tabular}
代过程如图 5 所示.

表 1 式(14)(15)应用于不同方法所得简支梁优化结构 


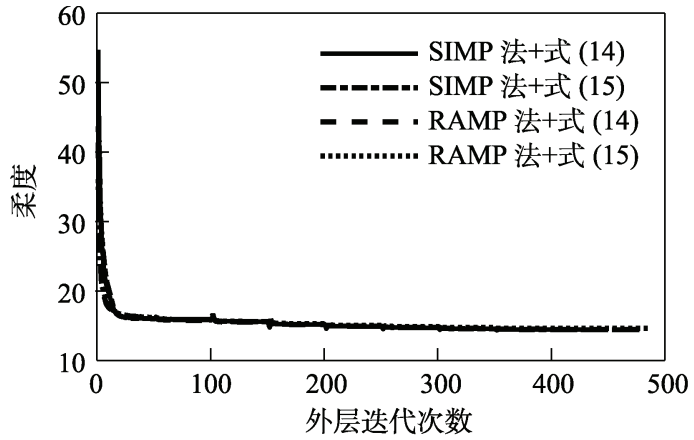

a. 总迭代详图

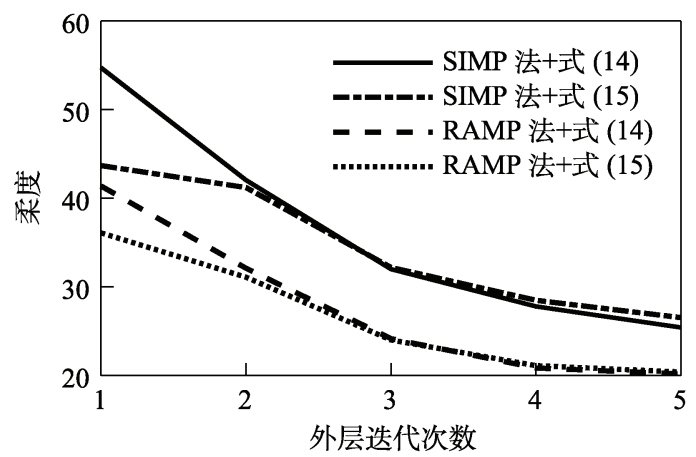

b. 始端局部放大图

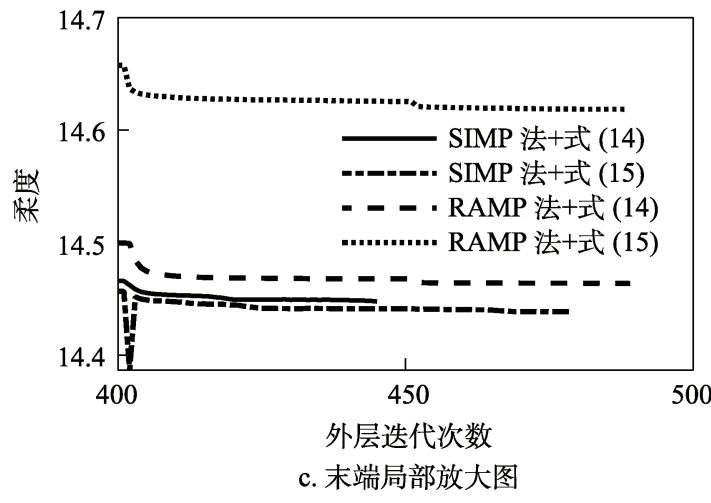

图 5 简支梁的柔度迭代过程
由表 1 和图 5 可知:

（1）上述 4 种方法中, SIMP 法和本文提出的式 (15)所得结构最优, 柔度仅 14.4385, 小于式(14), 所以，式(15)改进效果显著.

(2) 采用式(15)时, 第 1 次迭代所得初始柔度 低于式(14).

为了详细分析投影函数对 SIMP 和 RAMP 插 值方法的影响, 对单元密度在各区间的分布情况 进行统计, 统计数据如表 2 所示.

表 2 式(14)(15)应用于不同方法所得简支梁的 单元密度分布

\begin{tabular}{crrrrr}
\hline 单元密度 & \multicolumn{2}{c}{ SIMP 法 } & & \multicolumn{2}{c}{ RAMP 法 } \\
\cline { 2 - 3 } \cline { 5 - 6 } 区间 & 式(14) & 式(15) & & 式(14) & 式(15) \\
\hline $0.0 \sim 0.1$ & 3526 & 3566 & & 3586 & 3550 \\
$0.1 \sim 0.2$ & 0 & 0 & & 4 & 4 \\
$0.2 \sim 0.3$ & 16 & 0 & & 2 & 22 \\
$0.3 \sim 0.4$ & 4 & 0 & & 8 & 14 \\
$0.4 \sim 0.5$ & 24 & 0 & & 0 & 0 \\
$0.5 \sim 0.6$ & 0 & 2 & & 6 & 16 \\
$0.6 \sim 0.7$ & 56 & 32 & & 0 & 16 \\
$0.7 \sim 0.8$ & 40 & 52 & & 4 & 10 \\
$0.8 \sim 0.9$ & 62 & 40 & & 6 & 26 \\
$0.9 \sim 1.0$ & 3472 & 3508 & & 3584 & 3542 \\
$0.1 \sim 0.9$ & 202 & 126 & 30 & 108 \\
\hline
\end{tabular}

由表 2 可知, 式(15)对 SIMP 法所得结构中的 灰度单元有显著的抑制效果，而且 $0.1 \sim 0.5$ 的灰度 单元数量为 0 .

若仅改变 Heaviside 投影函数, 其他参数保持 不变, 2 种 Heaviside 投影函数所得优化结构如表 3 所示.

表 3 式(16)(17)应用于不同方法所得优化结构的对比

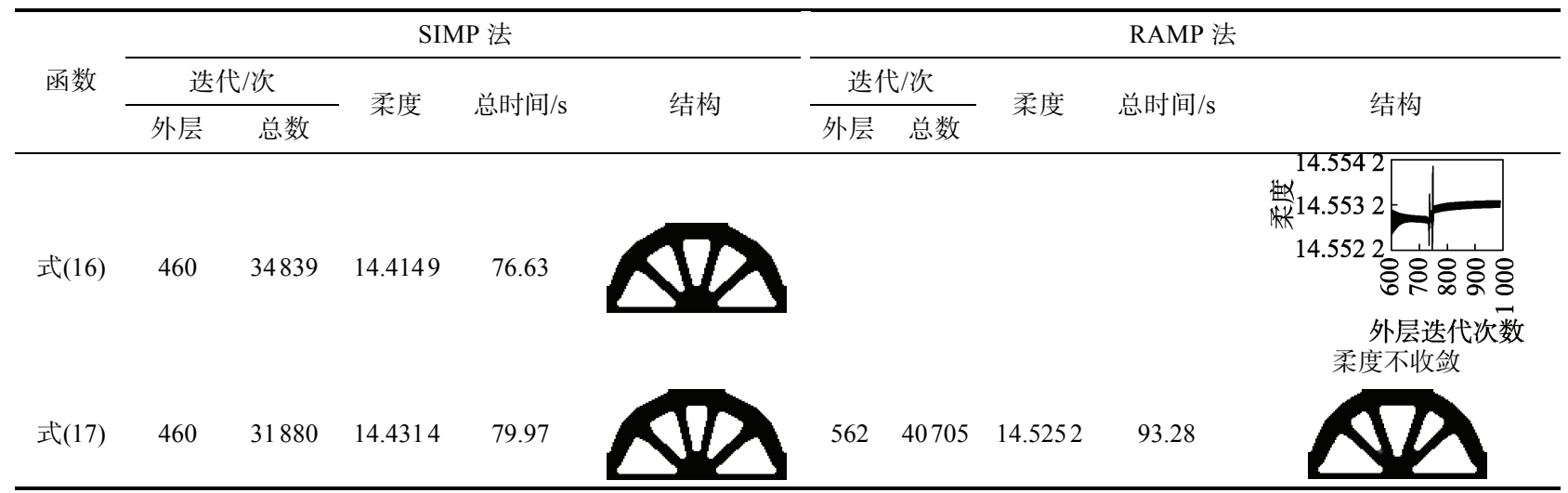

由表 3 可知:

（1）当同时采用 RAMP 法和式(16)时，算法不 收敛, 式(17)收敛性较好.

（2）采用 RAMP 法时，式(16)所得结构的柔度
仅可达到 14.5542, 大于式(17)所得结构的柔度 14.5252 , 式(17)改进效果显著.

\section{2 二维悬臂梁的拓扑优化}

悬臂梁左端固定, 离散单元个数为 $120 \times 50$, 
右下角承受集中力 $F$. 该悬臂梁的结构示意图如图 6 所示.

设 $F=1, E_{\min }=1 \times 10^{-9}, E_{0}=1, f=0.50, r_{\min }=2.5$, $p=3, q=3$; 式(14)(15)这 2 种投影函数所得优化 结构如表 4 所示, 柔度的计算迭代过程如图 7 所示.

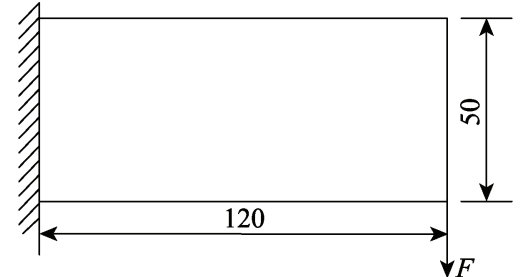

图 6 二维悬臂梁的结构示意图

表 4 式(14)(15)应用于不同方法所得悬臂梁优化结构的对比

\begin{tabular}{|c|c|c|c|c|c|c|c|c|c|c|}
\hline \multirow{3}{*}{ 函数 } & \multicolumn{5}{|c|}{ SIMP 法 } & \multicolumn{5}{|c|}{ RAMP 法 } \\
\hline & \multicolumn{2}{|c|}{ 迭代/次 } & \multirow{2}{*}{ 柔度 } & \multirow{2}{*}{ 总时间/s } & \multirow{2}{*}{ 结构 } & \multicolumn{2}{|c|}{ 迭代/次 } & \multirow{2}{*}{ 柔度 } & \multirow{2}{*}{ 总时间/s } & \multirow{2}{*}{ 结构 } \\
\hline & 外层 & 总数 & & & & 外层 & 总数 & & & \\
\hline 式(14) & 509 & 22112 & 105.6535 & 68.82 & & 464 & 20308 & 103.6330 & 66.38 & \\
\hline 式(15) & 393 & 17047 & 104.7079 & 54.11 & & 464 & 20336 & 103.5706 & 65.91 & \\
\hline
\end{tabular}

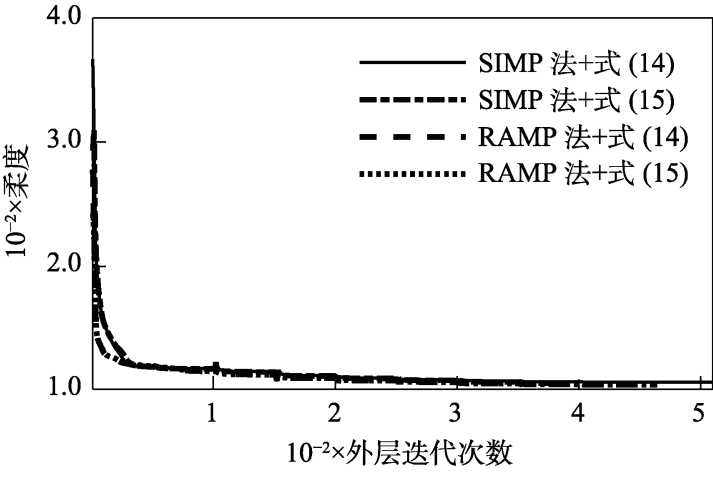

a. 总迭代详图

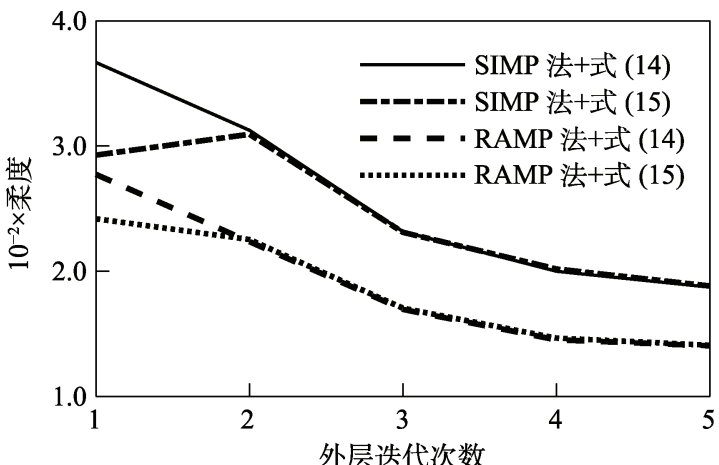

b. 始端局部放大图

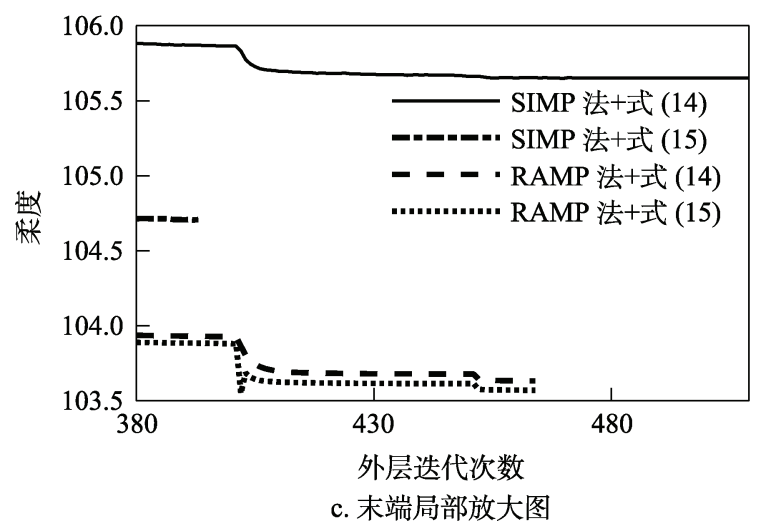

图 7 悬臂梁的柔度迭代过程
由表 4 和图 7 可知:

(1) 无论采用 SIMP 法还是 RAMP 法, 式(15) 所得柔度均小于式(14).

(2) 采用 SIMP 法时, 式(15)迭代次数少于式 (14)，其收敛更快.

（3）采用式(15)时，第 1 次迭代所得初始柔度 低于式(14).

为了详细分析投影函数对 SIMP 和 RAMP 插 值方法的影响, 对单元密度在各区间的分布情况 进行统计数据如表 5 所示.

表 5 式(14)(15)应用于不同方法所得悬臂梁的 单元密度分布

\begin{tabular}{lrrrrr}
\hline \multirow{2}{*}{$\begin{array}{c}\text { 单元密度 } \\
\text { 区间 }\end{array}$} & \multicolumn{2}{c}{ SIMP 法 } & & \multicolumn{2}{c}{ RAMP 法 } \\
\cline { 2 - 3 } \cline { 5 - 6 } 式(14) & 式(15) & & 式(14) & 式(15) \\
\hline $0.0 \sim 0.1$ & 2897 & 2920 & 2982 & 2992 \\
$0.1 \sim 0.2$ & 24 & 2 & 5 & 1 \\
$0.2 \sim 0.3$ & 23 & 39 & 4 & 3 \\
$0.3 \sim 0.4$ & 15 & 3 & 5 & 1 \\
$0.4 \sim 0.5$ & 8 & 4 & 7 & 0 \\
$0.5 \sim 0.6$ & 27 & 10 & 5 & 1 \\
$0.6 \sim 0.7$ & 36 & 18 & 1 & 8 \\
$0.7 \sim 0.8$ & 52 & 36 & 2 & 1 \\
$0.8 \sim 0.9$ & 77 & 153 & 2 & 4 \\
$0.9 \sim 1.0$ & 2841 & 2815 & 2987 & 2989 \\
$0.1 \sim 0.8$ & 185 & 112 & 29 & 15 \\
\hline
\end{tabular}

由表 5 可知, 无论采用 SIMP 法还是 RAMP 法, 本文式(15)所得结构中 $0.1 \sim 0.8$ 的灰色单元的数目 均小于式(14), 改进效果显著. 


\section{3 三维简支梁的拓扑优化}

悬臂梁左端面固定，右端下边缘中心承受集 中力, 离散单元个数为 $40 \times 15 \times 10$. 该三维悬臂梁 的结构示意图如图 8 所示.

设 $F=1, E_{\min }=1 \times 10^{-9}, E_{0}=1, f=0.17, r_{\min }=1.3$, $p=3, q=3$; 参数 $\beta$ 的初始值均为 1 , 每 3 步乘以 2 , 且 $\beta$ 的最大值为 512. 式(14)(15)所得优化结构如 表 6 所示，柔度的计算迭代过程如图 9 所示.

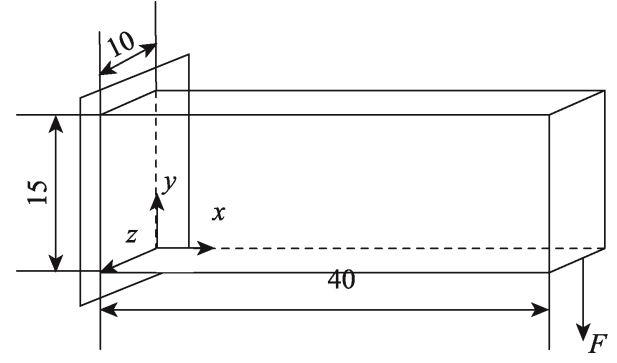

图 8 三维悬臂梁的结构示意图

表 6 式(14)(15)所得三维悬臂梁优化结构

\begin{tabular}{|c|c|c|c|c|c|c|c|c|c|c|}
\hline \multirow{3}{*}{ 函数 } & \multicolumn{5}{|c|}{ SIMP 法 } & \multicolumn{5}{|c|}{ RAMP 法 } \\
\hline & \multicolumn{2}{|c|}{ 迭代/次 } & \multirow{2}{*}{ 柔度 } & \multirow{2}{*}{ 总时间 $/ \mathrm{s}$} & \multirow{2}{*}{ 结构 } & \multicolumn{2}{|c|}{ 迭代/次 } & \multirow{2}{*}{ 柔度 } & \multirow{2}{*}{ 总时间 $/ \mathrm{s}$} & \multirow{2}{*}{ 结构 } \\
\hline & 外层 & 总数 & & & & 外层 & 总数 & & & \\
\hline 式(14) & 113 & 4799 & 44.7543 & 114.61 & & 160 & 6979 & 40.4627 & 146.49 & \\
\hline 式(15) & 104 & 4413 & 44.0105 & 105.24 & & 191 & 8344 & 40.5072 & 174.14 & \\
\hline
\end{tabular}

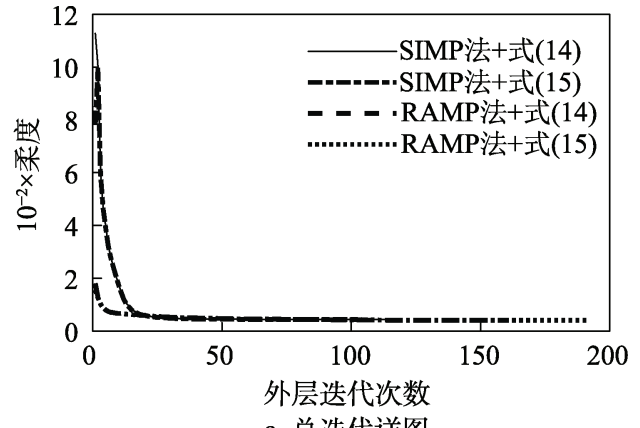

a. 总迭代详图

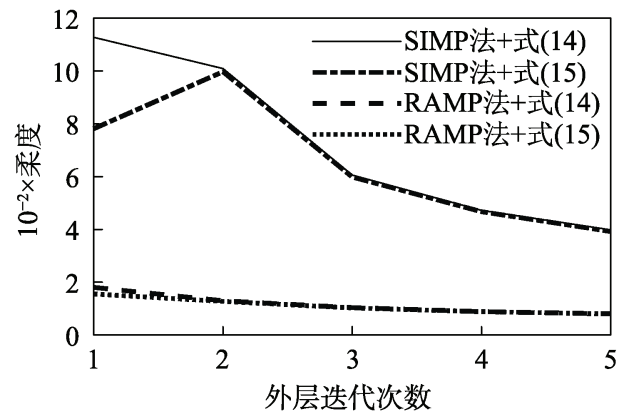

b. 始端局部放大图

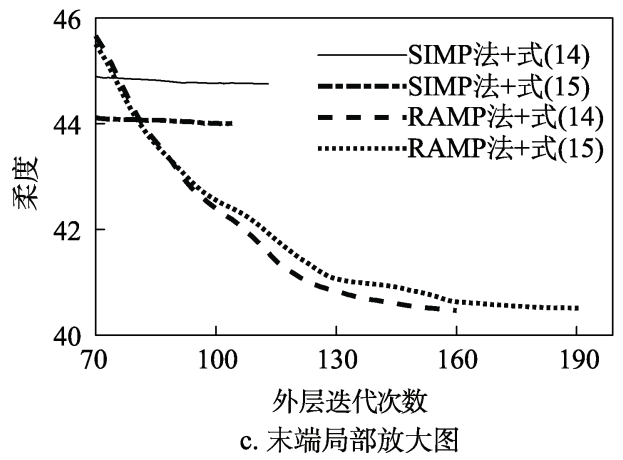

图 9 式(14)(15)优化三维悬臂梁的柔度迭代过程
由表 6 和图 9 可知，当采用 SIMP 法时，本文 提出的式(15)所得结构的柔度比式(14)小, 且本文 提出的式(15)迭代次数少于式(14), 改进效果显著.

为分析 4 种方法所得结构中灰度单元的数量, 对单元密度在各区间的分布情况进行统计, 统计 数据见表 7 .

表 7 式(14)(15)所得三维悬臂梁的单元密度分布

\begin{tabular}{crrrrr}
\hline 单元密度 & \multicolumn{2}{c}{ SIMP 法 } & & \multicolumn{2}{c}{ RAMP 法 } \\
\cline { 2 - 3 } \cline { 5 - 6 } & 式(14) & 式(15) & & 式(14) & 式(15) \\
\hline $0.0 \sim 0.1$ & 4826 & 4836 & & 4748 & 4744 \\
$0.1 \sim 0.2$ & 16 & 14 & 96 & 108 \\
$0.2 \sim 0.3$ & 38 & 48 & 78 & 90 \\
$0.3 \sim 0.4$ & 44 & 32 & 68 & 54 \\
$0.4 \sim 0.5$ & 46 & 54 & 56 & 50 \\
$0.5 \sim 0.6$ & 46 & 32 & 28 & 26 \\
$0.6 \sim 0.7$ & 32 & 24 & 18 & 12 \\
$0.7 \sim 0.8$ & 54 & 36 & 10 & 16 \\
$0.8 \sim 0.9$ & 72 & 64 & 18 & 22 \\
$0.9 \sim 1.0$ & 826 & 860 & 880 & 878 \\
$0.1 \sim 0.9$ & 348 & 304 & 372 & 378 \\
\hline
\end{tabular}

由表 7 可知，同时采用 SIMP 法和本文提出的 式(15)时, 所得结构中 $0.1 \sim 0.9$ 的灰度单元数目最少.

设参数 $\beta$ 的初始值均为 1 , 每 3 步乘以 2 , 且 $\beta$ 的最大值为 512 . 若将 Heaviside 投影函数改为 式(16)和式(17), 其他参数保持不变, SIMP 法可迅 
速收玫, 但 RAMP 法所得柔度变化较大. 根据多 次模拟所得结果可知: 若 $\beta$ 增加较快, 则 RAMP 法所得柔度波动较大, 算法的收玫性较差; 若 $\beta$ 增加较慢, 则 RAMP 法所得柔度较小, 但棋盘格 现象较明显.

设参数 $\beta$ 的初始值均为 1 , 每 3 步乘以 2 , 且 $\beta$ 的最大值为 512 . 当采用式(16)和式(17)时, SIMP 法所得优化结构见表 8 , 柔度的计算迭代过 程如图 10 所示. 由于 RAMP 法所得柔度波动较大, 算法收玫性较差，所以无法罗列其计算结果.

表 8 渐变 $\beta$ 的式(16)(17)所得三维简支梁优化结构

\begin{tabular}{llllll}
\hline 函数 & \multicolumn{2}{c}{ 迭代/次 } & 柔度 & 总时间 $/ \mathrm{s}$ \\
\cline { 5 - 6 } & 外层 & 总数 & & \\
式(16) & 27 & 1204 & 44.6266 & 30.78 \\
式(17) & 33 & 2926 & 44.4340 & 36.52
\end{tabular}

由表 8 和图 10 可知，当采用 SIMP 法时，虽然 本文提出的式(17)所得初始柔度较大，但迭代终止 时结构的柔度小于式(16), 改进效果显著.

若 $\beta$ 始终为 1 , 其他参数保持不变, 式(16)和 式(17)所得优化结构如表 9 所示, 柔度的计算迭代 过程如图 11 所示.
由表 9 和图 11 可知, 无论采用 SIMP 法还是 RAMP 法, 本文提出的式(17)所得结构的柔度均小 于式(16).

为分析 4 种方法所得结构中灰度单元的数量,

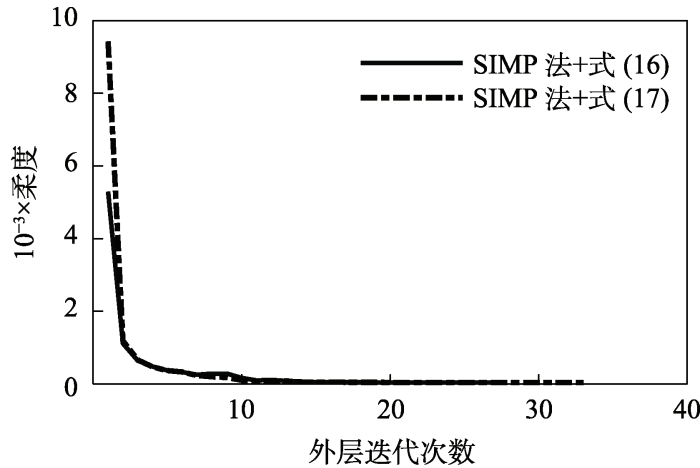

a. 总迭代详图

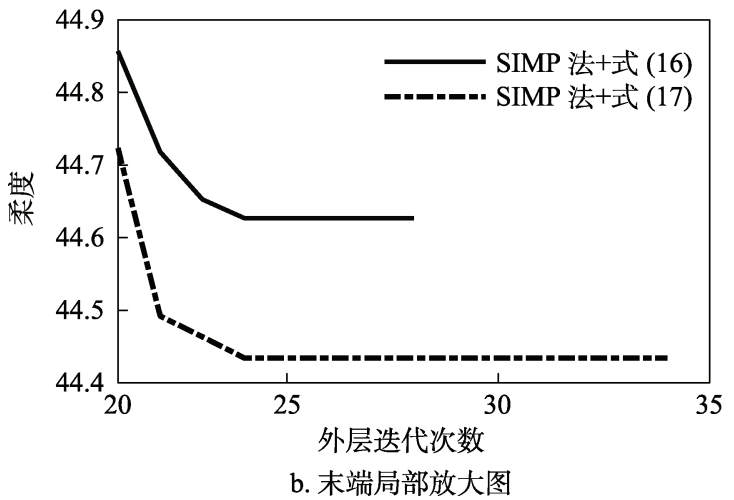

图 10 渐变 $\beta$ 的式(16)(17)优化三维悬臂梁 的柔度迭代过程

表 9 固定 $\beta$ 的式(16)(17)所得三维简支梁优化结构

\begin{tabular}{|c|c|c|c|c|c|c|c|c|c|c|}
\hline \multirow{3}{*}{ 函数 } & \multicolumn{5}{|c|}{ SIMP 法 } & \multicolumn{5}{|c|}{ RAMP 法 } \\
\hline & \multicolumn{2}{|c|}{ 迭代/次 } & \multirow{2}{*}{ 柔度 } & \multirow{2}{*}{ 总时间/s } & \multirow{2}{*}{ 结构 } & \multicolumn{2}{|c|}{ 迭代/次 } & \multirow{2}{*}{ 柔度 } & \multirow{2}{*}{ 总时间/s } & \multirow{2}{*}{ 结构 } \\
\hline & 外层 & 外层 & & & & 外层 & 总数 & & & \\
\hline 式(16) & 249 & 10671 & 57.7683 & 244.71 & & 300 & 13059 & 47.7844 & 354.90 & \\
\hline 式(17) & 268 & 11489 & 57.3533 & 267.83 & & 300 & 13105 & 47.1735 & 268.68 & \\
\hline
\end{tabular}
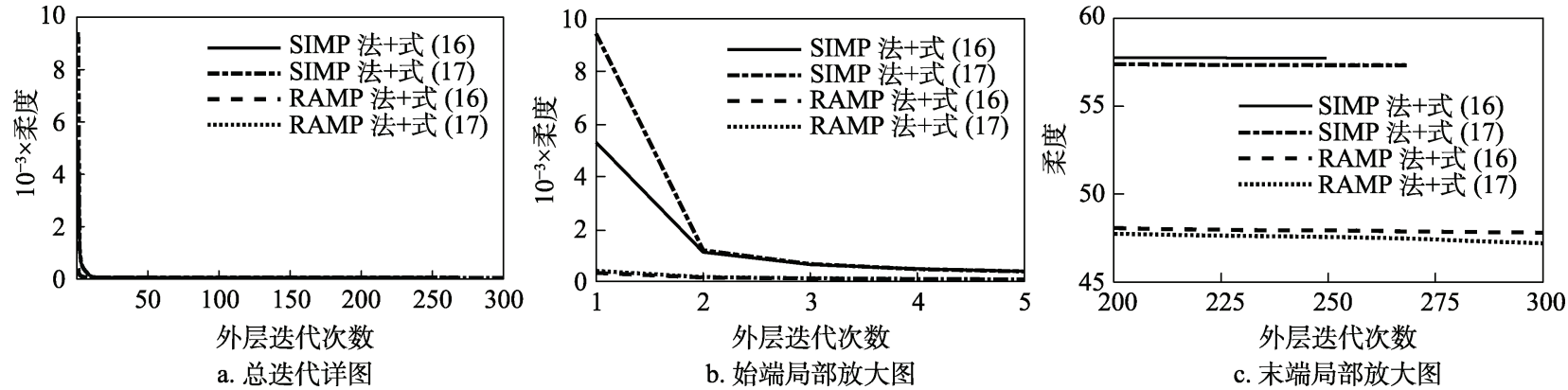

图 11 固定 $\beta$ 的式(16)(17)优化三维悬臂梁的柔度迭代过程 
对单元密度在各区间的分布情况进行统计，统计 数据见表 10 .

表 10 固定 $\beta$ 的式(16)(17)所得三维悬臂梁的 单元密度分布

\begin{tabular}{crrrrr}
\hline 单元密度 & \multicolumn{2}{c}{ SIMP 法 } & & \multicolumn{2}{c}{ RAMP 法 } \\
\cline { 2 - 3 } \cline { 5 - 6 } 区间 & 式(16) & 式(17) & & 式(16) & 式(17) \\
\hline $0.0 \sim 0.1$ & 4531 & 4466 & & 4420 & 4433 \\
$0.1 \sim 0.2$ & 298 & 356 & & 345 & 337 \\
$0.2 \sim 0.3$ & 55 & 16 & 151 & 77 \\
$0.3 \sim 0.4$ & 7 & 4 & 31 & 50 \\
$0.4 \sim 0.5$ & 23 & 22 & 30 & 35 \\
$0.5 \sim 0.6$ & 35 & 78 & 29 & 48 \\
$0.6 \sim 0.7$ & 147 & 250 & & 96 & 195 \\
$0.7 \sim 0.8$ & 227 & 120 & & 259 & 204 \\
$0.8 \sim 0.9$ & 473 & 442 & & 355 & 290 \\
$0.9 \sim 1.0$ & 204 & 246 & & 284 & 331 \\
$0.1 \sim 0.9$ & 1265 & 1288 & 1296 & 1236 \\
\hline
\end{tabular}

由表 10 可知，同时采用 RAMP 法和本文提出 的式(17)时, 所得结构中 $0.1 \sim 0.9$ 的灰度单元数目 最少. 此外, 无论采用 SIMP 法还是 RAMP 法, 应 用式(17)所得结构中 $0.1 \sim 0.8$ 的灰度单元数目均小 于式(16).

\section{4 结 语}

本文提出了一种改进的 Heaviside 投影函数, 为拓扑优化提供了一种抑制灰度单元的方法. 该 Heaviside 投影函数不仅可以求解二维和三维的拓 扑优化问题，而且可将其应用于 SIMP 和 RAMP 这 2 种插值模型. 通过 3 个算例说明了提出的 Heaviside 投影函数具有良好的通用性. 3 个算例的 计算结果表明:

（1）如果采用 SIMP 法, 式(15)所得结构的柔 度小于式(14), 改进效果显著.

（2） 2 个悬臂梁算例中，采用 SIMP 法时，式 (15)的收敛速度比式(14)更快.

(3) 当同时采用式(16)和 RAMP 法时, 算法存 在不收玫的情况. 因此, 式(17)收玫性较好.

(4) 三维悬臂梁算例中，同时采用式(17)和 RAMP 法时, 所得结构的柔度最小, 且灰度单元的 数量最少, 改进效果显著.

(5) 与式(14)(16)相比, 本文式(15)(17)中均有 且仅有一个密度的一次项，而式(14)(16)中包含 2 个密度的一次项。因此, 在密度更新时, 式(14) (16)需要调用 2 次单元密度矩阵, 而式(15)(17)仅需
调用 1 次单元密度矩阵, 所以调用单元密度矩阵的 次数减少了 $50 \%$.

\section{参考文献(References):}

[1] Bendsøe M P. Optimal shape design as a material distribution problem[J]. Structural Optimization, 1989, 1(4): 193-202

[2] Rietz A. Sufficiency of a finite exponent in SIMP (power law) methods $[\mathrm{J}]$. Structural and Multidisciplinary Optimization, 2001, 21(2): 159-163

[3] Stolpe M, Svanberg K. An alternative interpolation scheme for minimum compliance topology optimization[J]. Structural and Multidisciplinary Optimization, 2001, 22(2): 116-124

[4] Andreassen E, Clausen A, Schevenels M, et al. Efficient topology optimization in MATLAB using 88 lines of code[J]. Structural and Multidisciplinary Optimization, 2011, 43(1): 1-16

[5] Liu K, Tovar A. An efficient 3D topology optimization code written in Matlab[J]. Structural and Multidisciplinary Optimization, 2014, 50(6): 1175-1196

[6] Zhu B L, Zhang X M, Zhang H C, et al. Design of compliant mechanisms using continuum topology optimization: a review[J]. Mechanism and Machine Theory, 2020, 143: 103622

[7] Wei P, Li Z Y, Li X P, et al. An 88-line MATLAB code for the parameterized level set method based topology optimization using radial basis functions[J]. Structural and Multidisciplinary Optimization, 2018, 58(2): 831-849

[8] Yaghmaei M, Ghoddosian A, Khatibi M M. A filter-based level set topology optimization method using a 62-line MATLAB code[J]. Structural and Multidisciplinary Optimization, 2020, 62(2): 1001-1018

[9] Gao J, Luo Z, Xia L, et al. Concurrent topology optimization of multiscale composite structures in Matlab[J]. Structural and Multidisciplinary Optimization, 2019, 60(6): 2621-2651

[10] Liu H, Zong H M, Tian Y, et al. A novel subdomain level set method for structural topology optimization and its application in graded cellular structure design[J]. Structural and Multidisciplinary Optimization, 2019, 60(6): 2221-2247

[11] Fuchs M B, Jiny S, Peleg N. The SRV constraint for 0/1 topological design[J]. Structural and Multidisciplinary Optimization, 2005, 30(4): 320-326

[12] Wu Yifan, Zheng Bailin, He Lyuyang, et al. Equivalent conversion method of gray-scale elements for SIMP in structures topology optimization[J]. Journal of Computer-Aided Design \& Computer Graphics, 2017, 29(4): 759-767(in Chinese) (吴一帆, 郑百林, 何旅洋, 等. 结构拓扑优化变密度法的灰 度单元等效转换方法 $[\mathrm{J}]$. 计算机辅助设计与图形学学报, 2017, 29(4): 759-767)

[13] Zhang Yifei. Research on structural topology optimization method based on PTO algorithm[D]. Xi'an: Xi'an University of Electronic Science and Technology, 2018(in Chinese) (张逸飞. 基于 PTO 算法的结构拓扑优化方法研究[D]. 西 安: 西安电子科技大学, 2018)

[14] Xu Xiaokui, Guo Baofeng, Jin Miao. Structural topology optimization based on density-volume interpolation scheme[J]. China Mechanical Engineering, 2017, 28(11): 1269-1273(in 
Chinese)

(许小奎, 郭宝峰, 金沝. 基于密度体积插值方法的结构拓 扑优化[J]. 中国机械工程, 2017, 28(11): 1269-1273)

[15] Fu Yongqing, Zhang Xianmin. Multi-level evolutionary approach for the black-and-white topology optimization de$\operatorname{sign}[\mathrm{J}]$. China Mechanical Engineering, 2017, 28(11): 1292-1299(in Chinese)

(付永清, 张宪民. 多层渐进黑白拓扑优化设计方法 [J]. 中 国机械工程, 2017, 28(11): 1292-1299)

[16] Du Yixian, Fu Junjian, Yan Shuangqiao, et al. Application of compactly supported Shepard approximation in topology optimization[J]. Journal of Huazhong University of Science and Technology: Nature Science Edition, 2013, 41(8): 101-105(in Chinese)

(杜义贤, 付君健, 严双桥, 等. 紧支 Shepard 近似在拓扑优 化中的应用研究 [J]. 华中科技大学学报: 自然科学版, 2013, 41(8): 101-105)

[17] Guest J K, Prévost J H, Belytschko T. Achieving minimum length scale in topology optimization using nodal design variables and projection functions[J]. International Journal for Numerical Methods in Engineering, 2004, 61(2): 238-254

[18] Sigmund O. Morphology-based black and white filters for topology optimization[J]. Structural and Multidisciplinary Optimization, 2007, 33: 401-424

[19] Long Kai, Zhao Hongwei. A modified optimality criterion method for gray elements suppression[J]. Journal of Computer-Aided Design \& Computer Graphics, 2010, 22 (12): 2197-2201(in Chinese)

(龙凯, 赵红伟. 抑制灰度单元的改进优化准则法 $[\mathrm{J}]$. 计算
机辅助设计与图形学学报, 2010, 22(12): 2197-2201)

[20] Zhao W C, Chen L L, Zheng C J, et al. Design of absorbing material distribution for sound barrier using topology optimization[J]. Structural and Multidisciplinary Optimization, 2017, 56(2): 315-329

[21] Qin Haoxing, An Zongwen, Sun Daoming. Improved guide-weight method on solving topology optimization problems and gray-scale filtering method[J]. Journal of Computer-Aided Design \& Computer Graphics, 2015, 27(10): 2001-2007(in Chinese)

(秦浩星, 安宗文, 孙道明. 改进的导重法求解拓扑优化问 题及灰度过滤技术 [J]. 计算机辅助设计与图形学学报, 2015, 27(10): 2001-2007)

[22] Lee D, Shin S. Topological optimal material design of structures with moved and regularized Heaviside function[J]. Iranian Journal of Science and Technology, Transactions of Mechanical Engineering, 2020, 44(1): 103-117

[23] Du Yixian, Zhang Yan, Li Hanzhao, et al. Topology optimization of node density interpolation method with polarization properties[J]. Journal of Machine Design, 2018, 35(3): 80-85(in Chinese)

(杜义贤, 张严, 李涵钊, 等. 具有极化特性的拓扑优化节点 密度插值方法 [J]. 机械设计, 2018, 35(3): 80-85)

[24] Li Hao. Research on topological optimization method of continuum structure based on variable density method[D]. Wuhan: Huazhong University of Science and Technology, 2011: 17-18 (in Chinese)

(李好. 基于变密度法的连续体结构拓扑优化方法研究 [D]. 武汉: 华中科技大学, 2011: 17-18) 